\title{
Anomalous processing in schizophrenia suggests adaptive event-action coding requires multiple executive brain mechanisms.
}

\author{
Robert D. Oades and Katya Kreul
}

\author{
Behavioral and Brain Sciences (2001), 24, 895-896
}

DOI:10.1017/S0140525X0138010X

Biopsychology Group, Clinic for Child and Adolescent Psychiatry, University of Essen, Virchowstr. 174, 45147 Essen, Germany. Email: oades@uni-essen.de

Commentary on : Hommel, Müsseler, Aschersleben \& Prinz :

"The theory of event coding (TEC):

A framework for perception and action planning"

Behavioral and Brain Sciences (2001), 24, 849-937 (Abstract at the end)

\begin{abstract}
:
The integration of perceived events with appropriate action usually requires more flexibility to result in adaptive responses than Hommel et al report in their selective review. The need for hierarchies of function that can intervene and the existence of diverse mediating brain mechanisms can be illustrated by the non-adaptive expression in psychiatric illness of negative priming, blocking, and affective responses.
\end{abstract}

\section{Commentary:}

Representatives of each arm of the firm gather around the conference table: there are those who interact with the outside world, the product makers and the salesmen, as well as those who organize their work, the production managers and market researchers. Not missing, of course are those with an overview over large parts if not all the firm's activities, namely, various directors ('executives'). We generalize about the function of each of these representatives, although in fact some have more abilities than others, and most are young enough to acquire more skills.

Hommel et al. are critical of Sanders (1983: p.7) chart which stretches from stimulus processing to motor adjustment (interactions with the outside world), and flows over feature extraction and response choice in the hierarchy of synthesis and rationalization (organization and executive management). But they seem never to be quite able to break free from this historical precedent. The metaphor of the firm still seems to apply.

It is important for Hommel et al., for example, that the binding of features and knowledge via selective attention to make an object file (Kahneman et al. (1992, p. 29 ) is the sort of psychological process operating in the perceptuo-motor interface which renders a distinction between perception and action functionally irrelevant. Though perhaps an anatomical distinction might still be tenable. Does it matter whether our metaphorical conference actually occurs around a table at one locus or functions thanks to a software-based network between topographically distributed participants? We think it does not usually matter. Indeed, in a similar vein the authors cite approvingly indications that gamma oscillations seem to reflect binding functions even between disparate sensory and motor areas (Roelfsema et al. 1997). 
We made no mention of a specific product for the firm: for some products there may be roles or functions that are best united in one capacity, for instance, in product management and market research, just as neurophysiologically speaking they could be united in the functions of a "mirror neurone" receptive to input about the sorts of motor action in whose initiation it also participates (Gallese et al. 1996, p. 12). But are these not exceptions to the usual organizing principles?

There may be a sense in which the TEC can be tested, an aspect about which Hommel et al. were pessimistic (p.5), if one does not neglect the anatomy and the mechanisms (cf. numerous versions of short/long feedback loops and reafference: e.g. the monitoring of action in fig 5.1 of Frith 1992). Hommel et al state, "Not only should object and event perception be mediated by object-file types of structures, but action planning should be mediated by the very same entities as well (... 'action files')"; [p. 31]. Problems that concern some psychiatric patients (schizophrenia, obsessive compulsive disorder, OCD) can involve situations crucial to stages in the development of the TEC argument. The following three example on the influence of irrelevant stimulation in mental illness, in priming and blocking, and in the prediction of the consequences, illustrate the differentiation of central executive mechanisms rather than their unity, as required by the Theory of Event Coding.

First, negative priming (NP) is said to provide pivotal evidence for the paradigmatic construction of an object file from subordinate perceptual features [pp.29-30] applicable to the integration of attention and intention. An interruption of the normal interference of the prime (e.g. in OCD: Enright \& Beech, 1993), would appear well suited to explain checking compulsions. But in fact checking does not relate to impaired inhibition in NP, but rather to facilitation on positive priming trials (Ferraro et al. 1996; Hartston \& Swerdlow 1999). It seems counterintuitive to expect that a correctly assembled object-action file should enhance the pathology without recourse to re-modeling several components of the process and their interactions. Interestingly, in the normal population impaired NP may relate more to schizotypal features (Sturgill \& Ferraro, 1997).

NP is impaired in acutely ill patients with positive symptoms of schizophrenia (Peters et al. 2000; Williams 1996), but is otherwise present in the disorganized and those with negative symptoms. This brings us to the second point. Hommel et al. [p.17] write that although actions performed in sympathy can involve involuntary automatic processes, they are often intentional responses to a situation that reflect object-action plan integration. We would predict that if NP (and the all important binding mechanisms, Haig et al. 2000) are impaired in positive schizophrenia, then inappropriate affect and sympathy should be a feature of these patients: which is not the usual finding. In fact, inappropriate affect is a cardinal symptom of the 'disorganized' type of schizophrenia that may, but often does not involve the primary symptoms of positive schizophrenia (Straube \& Oades 1992).

Our third point concerns Hommel et al.'s report that the direction taken by a masked arrow was less accurately reported when it concurred with left or right key responses than in the mismatch condition. Rather than representing an example of the influence of action plans on perception, we believe this may be described better as an example of conditioned blocking. That is to say training with (the conditioning of) one 
response makes it less likely that the wholly redundant information associated with another stimulus is acquired. (The arrow is superfluous, the direction it points is not or poorly registered for recall.) This is an entirely attention-related phenomenon seen in many forms of learning. It is also important to note that the details of the brain mechanisms involved in blocking differ from those associated with NP. This is reflected by reports that patients with a negative or nonparanoid forms of schizophrenia are impaired on blocking (Bender et al. 2001 Oades et al 1996), not those with the positive form of the illness as in NP.

We conclude that it may be the exception rather than the rule that object files representing the binding of attentionally derived information achieve the functional identity of action files. Our argument is based on the different problems encountered by patients with one or another form of mental illness, and this implies the differential ('executive') intervention of the brain mechanisms involved in each of these phenomena. The impairments resulting from brain damage or psychopathology point to the diversity of these components and the need for anatomically diverse executive mechanisms often based in the frontal lobes, to provide the link(s) to appropriate action plans.

\section{References}

Bender, S., Müller, B., Oades, R. D. \& Sartory, G. (2001). Conditioned blocking and schizophrenia: a replication and study of the role of symptoms, age, onset-age of psychosis and illnessduration. Schizophrenia Research, 49:157-170.

Ferraro, F. R., Jones, D. \& McManus, G. (1996) Relationship of Maudsley obsessive-compulsive inventory and negative priming: some preliminary evidence. Brain and Cognition, 32:284285

Enright, S. J. \& Beech, A. R. (1993). Further evidence of reduced cognitive inhibition in obsessive-compulsive disorder. Personality and Individual Differences, 14:387-395.

Frith, C. D. (1992). The cognitive neuropsychology of schizophrenia. Lawrence Erlbaum Associates.

Gallese, V., Fadiga, L., Fogassi, L. \& Rizzolatti, G. (1996) Action recognition in the premotor cortex. Brain, 119:593609.

Haig, A. R., Gordon, E., De Pascalis, V., Meares, R. A., Bahramali, H. \& Harris, A. (2000) Gamma activity in schizophrenia: evidence of impaired network binding? Clinical Neurophysiology, 111:1461-1468.

Hartston, H. J. \& Swerdlow, N. R. (1999). Visuospatial priming and Stroop performance in patients with obsessive compulsive disorder. Neuropsychology, 13:447-457.

Kahneman, D., Treisman, A. M. \& Gibbs, B. (1992). The reviewing of object files. Object-specific integration of information. Cognitive Psychology, 24:175219.

Oades, R. D., Zimmermann, B. \& Eggers, C. (1996) Conditioned blocking in patients with paranoid, non-paranoid psychosis or obsessive compulsive disorder: associations with symptoms, personality and monoamine metabolism. Journal of Psychiatric Research, 30: 369-390.

Peters, E. R., Pickering, A. D., Kent, A., Glasper, A., Irani, M., David, A. S., Day, S. \& Hemsley, D. R. (2000). The relationship between cognitive inhibition and psychotic symptoms. Journal of Abnormal Psychology, 109:386-395.

Roelfsema, P. R., Engel, A. K., Koenig, P. \& Singer, W. (1997). Visuomotor integration is associated with zero 
time-lag synchronization among cortical areas. Nature, 385:157-161.

Sanders, A. F. (1983). Towards a model of stress and human performance. Acta Psychologica, 53:61-97.

Straube, E. R. \& Oades, R. D. (1992).

Schizophrenia: empirical research and findings. Academic Press.
Sturgill, D. S. \& Ferraro, F. R. (1997). Predicting negative priming: effects of stereotypy, cognitive failures and anxiety. Personality and Individual Differences, 23:291-304.

Williams, L. M. (1996). Cognitive inhibition and schizophrenic symptom subgroups. Schizophrenia Bulletin, 22:139-151.

\title{
Target article abstract:
}

The theory of event coding (TEC): A framework for perception and action planning

$$
\text { Bernhard Hommel }{ }^{1,2} \text { Jochen Müsseler }{ }^{2} \text {, Gisa Aschersleben }{ }^{2} \text { and Wolfgang Prinz }{ }^{2}
$$

Behavioral and Brain Sciences (2001), 24, 849-937 - DOI:10.1017/S0140525X01000103

\footnotetext{
${ }^{1}$ Section of Experimental and Theoretical Psychology, University of Leiden, 2300 RB Leiden, The Netherlands hommel@fsw.leidenuniv.nl

${ }_{2}$ Max Planck Institute for Psychological Research, D-80799 Munich, Germany muesseler@mpipfmuenchen.mpg.de aschersleben@mpipf-muenchen.mpg.de prinz@mpipf-muenchen.mpg.de www.mpipfmuenchen.mpg.de/ prinz
}

\begin{abstract}
Traditional approaches to human information processing tend to deal with perception and action planning in isolation, so that an adequate account of the perception-action interface is still missing. On the perceptual side, the dominant cognitive view largely underestimates, and thus fails to account for, the impact of action-related processes on both the processing of perceptual information and on perceptual learning. On the action side, most approaches conceive of action planning as a mere continuation of stimulus processing, thus failing to account for the goal-directedness of even the simplest reaction in an experimental task. We propose a new framework for a more adequate theoretical treatment of perception and action planning, in which perceptual contents and action plans are coded in a common representational medium by feature codes with distal reference. Perceived events (perceptions) and to-be-produced events (actions) are equally represented by integrated, tasktuned networks of feature codes - cognitive structures we call event codes. We give an overview of evidence from a wide variety of empirical domains, such as spatial stimulusresponse compatibility, sensorimotor synchronization, and ideomotor action, showing that our main assumptions are well supported by the data.
\end{abstract}

